\title{
Thrombus Formation and Platelet-Vessel Wall Interaction in the Nephrotic Syndrome under Flow Conditions
}

\author{
Jaap Jan Zwaginga, ${ }^{*}$ Hein A. Koomans, ${ }^{*}$ Jan J. Sixma, ${ }^{\star}$ and Ton J. Rabelink \\ Departments of * Nephrology and ${ }^{\ddagger}$ Hematology, University Hospital Utrecht, 3508 GA Utrecht, The Netherlands
}

\begin{abstract}
Increased in vitro platelet aggregability and hypercoagulability are generally held to be main determinants in the prethrombotic state in nephrosis. In vivo, however, thrombotic events depend on the dynamic interaction of flowing blood with the vessel wall. The present study confirms that aggregability of platelets of nephrotic patients is significantly increased by mere stirring or by exogenous stimuli as adenosine diphosphate and arachidonic acid. Moreover, the nephrotic patients have high von Willebrand factor and decreased red blood cell deformability, which normally increase platelet-vessel wall interaction. However, perfusion studies under well-defined flow conditions, in which anticoagulated nephrotic blood was exposed to deendothelialized human umbilical artery segments and sprayed collagen, showed normal platelet adhesion and only a modest increase in the deposition of platelet aggregates. This suggests that some factor counteracts platelet-vessel wall interaction under flow conditions in the nephrotic syndrome. When tissue factor associated with endothelial extracellular matrix (ECM) was allowed to generate thrombin, perfusions with nephrotic blood over this ECM resulted in a strong increase in fibrin generation. The capacity of patient blood to form increased amounts of fibrin appeared strongly correlated with the level of hyperfibrinogenemia. Platelet adhesion as well as aggregation in these experiments was even decreased below control values. This suggests that fibrin coverage may block the direct contact between blood platelets and matrix. We therefore also studied the isolated effect of high fibrinogen on platelet-vessel wall interaction by increasing fibrinogen concentrations in normal blood. Modulation of fibrinogen concentrations in normal blood could mimic all the observations in nephrotic blood: platelet aggregation in suspension increased with increasing concentrations of fibrinogen, while platelet adhesion and aggregate formation under flow conditions decreased. In perfusions over tissue factor-rich matrix, fibrin deposition increased. Therefore, our observations indicate that nephrotic hyperaggregability in suspension is not associated with increased platelet vessel wall-interaction under flow conditions. The latter is probably counteracted by high levels of fibrinogen. Our observations further suggest that hyperfibrinogenemia may be a major thrombotic risk factor in nephrosis by inducing more fibrin depositions. (J. Clin. Invest. 1994. 93:204-211.) Key words: fibrino-
\end{abstract}

Address correspondence to Dr. A. J. Rabelink, Department of Nephrology and Hypertension (F03.226), University Hospital Utrecht, P.O. Box 85500, 3508 GA Utrecht, The Netherlands.

Received for publication 12 May 1993 and in revised form $16 \mathrm{Au}$ gust 1993.

J. Clin. Invest.

(C) The American Society for Clinical Investigation, Inc.

0021-9738/94/01/0204/08 \$2.00

Volume 93, January 1994, 204-211 gen - hypercoaguability - nephrotic syndrome - platelets • subendothelium

\section{Introduction}

The risk for acute thromboembolic complications in patients with a nephrotic syndrome is unequaled in clinical practice and generally attributed to combined abnormalities in platelet and coagulation-dependent hemostasis $(1,2)$. Hypercoaguability may also contribute to the development of atherosclerosis (3) and glomerular injury (4), other well-known complications of the nephrotic syndrome. Various laboratory abnormalities in nephrosis may play a role in the prethrombotic state: hyperfibrinogenemia $(5)$, increased levels of clotting factors $(5,6)$, decreased levels of antithrombin III (7), decreased fibrinolytic potential (5), and enhanced aggregability of platelets suspended in plasma $(8-11)$.

However, these laboratory abnormalities are hard to extrapolate to in vivo hemostasis. Studies with perfusion systems, which allow the dynamic interaction of flowing blood with (injured) vessel wall, have shown that the hemostatic mechanisms are for a large part flow regulated (12). Higher blood flow (shear rate) promotes the transport of platelets towards the vessel wall by red blood cells and therefore platelet-dependent hemostasis. Higher platelet count, hematocrit, and increased red blood cell rigidity add to the efficiency of this platelet transport $(13,14)$. Shear rate also, modulates subsequent platelet binding to adhesive molecules deposited or present at the injured vessel wall, e.g., von Willebrand factor (vWF) (15). Coagulation-dependent hemostasis is initiated by vessel wallassociated tissue factor and involves thrombin-induced fibrin deposition and platelet aggregation. Again, shear rate regulates these processes (16).

Thus far, the influence of flow on hemostasis in the nephrotic syndrome has not been taken into account. Therefore, whole blood of nephrotic patients and normal control donors was perfused over models of injured vessel wall in well-characterized perfusion chambers. First, citrate-anticoagulated whole blood was perfused over deendothelialized human umbilical artery segments and over sprayed collagen (fibrillar type I). Both surfaces induce platelet adhesion as well as platelet aggregation; collagen as one of the constituents of the deeper vessel wall, however, is a more potent stimulus of platelet aggregation (17). In this way we determined that increased vWF levels and red blood cell rigidity and the in vitro hyperaggregability in nephrosis do not coincide with an increased platelet-vessel wall interaction under physiologic flow conditions. Second, low molecular weight heparin ( LMWH) ${ }^{1}$ was used as anticoagulant in additional perfusions. Where LMWH inhibits coagula-

1. Abbreviations used in this paper: AT III, antithrombin III; ECM, extracellular matrix; FPA, fibrinopeptide A; LMWH, low molecular weight heparin; PRP, platelet-rich plasma. 
tion during in vitro handling of the blood, it does allow thrombin generation by tissue factor associated with extracellular matrix of cultured human umbilical vein endothelial cells (ECM). The prothrombin-thrombin transition during perfusions can be monitored by measuring the release of peptide fragment $1+2$ from prothrombin in the perfusate. Hypercoagulability in nephrosis was illustrated by increased fibrin generation (monitored by thrombin-dependent fibrinopeptide-A [FPA] release from fibrinogen) and fibrin deposition on the perfused matrix. Fibrinogen as platelet-binding and -interlinking protein and as substrate of thrombin-induced fibrin formation is almost invariably increased in nephrotic patients $(1,2)$. In perfusions with control blood in which fibrinogen was artificially raised we could further substantiate that nephrotic hyperfibrinogenemia may play a major regulatory role in hemostasis and thrombogenesis.

\section{Methods}

Patients. Studies were performed in two groups of 10 nephrotic patients (characteristics in Table I) without important loss of renal function as estimated from creatinine clearance $(92 \pm 14 \mathrm{ml} / \mathrm{min}$ in group 1 and $91 \pm 13 \mathrm{ml} / \mathrm{min}$ in group 2). Blood of patients in group 1 was used in platelet aggregation studies, in red blood cell deformability measurements, and in citrate perfusions to substantiate within the same patient the effect of in vitro abnormalities on actual platelet-dependent hemostasis. The blood of patients in group 2 was used for measurements of clotting factors and for $\mathrm{LMWH}$ perfusions ( vide infra) to relate coagulation abnormalities to coagulation-dependent thrombus formation during perfusions. Parallel to every experiment with patient blood, control experiments were performed with blood collected from healthy members from the medical staff. Daily variations in experimental conditions (e.g., reactivity of perfusion surface) were in this way accounted for. All patients and control subjects were without medication for at least $2 \mathrm{wk}$ before the study; they gave informed consent and the study was approved by the University Hospital Ethical Committee for Studies in Humans.

Red blood cell deformability. Red blood cell deformability was measured by a laser-diffractometer set-up, essentially as described in detail previously $(18,19)$. In this method laser light is diffracted by a red blood cell suspension. When shear stress (in the present study 30 $\mathrm{N} / \mathrm{m}^{2}$ ) is applied to the suspension and to the red blood cells, it will cause elongation of the diffraction pattern. This elongation is a measure for the actual shear-induced red blood cell deformation. Higher values indicate higher deformability of red blood cells. To study intrinsic red blood cell qualities, $15 \mu \mathrm{l}$ of EDTA ( $2 \mathrm{mM}$ )-anticoagulated whole blood was suspended in $2.25 \mathrm{ml}$ of $7 \%$ polyvinylpyrrolidone

\section{Table I. Patient Group Characteristics}

\begin{tabular}{lcc}
\hline & Group 1 & Group 2 \\
\hline Histological diagnosis (biopsy proved, $n$ ): & & \\
$\quad$ Membraneous glomerulopathy & 5 & 6 \\
$\quad$ Membranoproliferative glomerulonephritis & 1 & 1 \\
$\quad$ Mesangioproliferative glomerulonephritis & 2 & 1 \\
Focal glomerulosclerosis and hyalinosis & 2 & 1 \\
$\quad$ Minimal change glomerulopathy & & 1 \\
Male/female $(n)$ & $7 / 3$ & $8 / 2$ \\
Age range $(y r)$ & $18-56$ & $18-48$ \\
Proteinuria $(g / 24 h)$ & $7.6 \pm 3.9$ & $5.7 \pm 2.1$ \\
Plasma albumin $(g / l i t e r)$ & $19 \pm 4$ & $18 \pm 4$ \\
\end{tabular}

(PVP; 360,000 mol wt, Sigma Chemical Co., St. Louis, MO) in PBS ( $1: 10 \mathrm{vol} / \mathrm{vol} 0.1 \mathrm{M}$ sodium phosphate buffer, $\mathrm{pH} 7.4$, in $150 \mathrm{mMol}$ $\mathrm{NaCl}$ ). To study interaction of the nephrotic red cell with plasma components the PVP was diluted in autologous plasma $(50 \mathrm{mg} / \mathrm{ml})$. PVP presence resulting in a high viscosity of the medium was necessary to obtain shear stress values that will cause deformation of red cells.

Platelet aggregation in suspension. Blood from patients and controls was collected into $1: 10$ ( $\mathrm{vol} / \mathrm{vol}) 110 \mathrm{mM}$ trisodium citrate by clean venepuncture. Platelet-rich plasma (PRP) was prepared (10-min centrifugation at $150 \mathrm{~g}$ and $20^{\circ} \mathrm{C}$ ) and the platelet count was corrected to 200,000 / $\mu$ l with autologous platelet poor plasma (10-min centrifugation at $3,000 \mathrm{~g}$ and $20^{\circ} \mathrm{C}$ ). Within $2 \mathrm{~h}$ after blood collection, aggregation studies with this PRP were performed at $37^{\circ} \mathrm{C}$ in a PAP-4 aggregometer (Bio/Data, Hatboro, PA) at $900 \mathrm{rpm}$. AA (Bio/Data) in final concentrations of 1.5-, 0.75-, 0.375-, $0.250 \mathrm{mM}$ and ADP (Dade, Aquada, PA) in concentrations of 5-, 2.5-, 1.67-, $0.84 \mu \mathrm{M}$ were used as stimuli. In addition, spontaneous platelet aggregation was determined by the mere stirring of the PRP. To avoid interference by turbidity differences due to high plasma lipids, we determined platelet aggregation not by the change in light transmission but by directly counting single (not aggregated) platelets remaining in the PRP after 3 min of AA and ADP stimulation and after $15 \mathrm{~min}$ of mere stirring. Single platelet counts were determined by a platelet analyzer (model 810 , Baker Instruments, Allentown, PA) with size apertures set between 3.2 and $16 \mu \mathrm{m}^{3}$ as described by Verhoeven et al. (20) and expressed as percentage of initial platelet count.

Perfusion studies with citrated blood. Blood from patients and controls was collected into $1: 10$ ( $\mathrm{vol} / \mathrm{vol}$ ) $110 \mathrm{mM}$ trisodium citrate. To avoid that initial differences in hematocrit and platelet count between patient and control blood would influence adhesion, perfusates were standardized to a platelet count of 200,000 platelets / $\mu \mathrm{l}$ and a hematocrit of 0.40 . Standardized perfusates were obtained by adding autologous PRP, platelet poor plasma (PPP), and red blood cells (RBC) together in proper quantities. PRP, PPP, and RBC were obtained by centrifugation of whole blood.

Perfusions with steady flow were carried out in a modified annular perfusion chamber (21) adapted from the original described by Baumgartner (12) or with the double rectangular perfusion chamber developed by Sakkariassen et al. (22). In the annular perfusion chamber inverted deendothelialized artery segments isolated from human umbilical cord were used as a model of injured vessel wall. Arteries were mounted on two separate central rods, which fitted in serially connected annular perfusion chambers. The rectangular perfusion chamber contained two pairs of Thermanox coverslips ( Lab-Tek Division, Miles Laboratories, Naperville, IL) with sprayed fibrillar equine collagen $\left(18.5 \mu \mathrm{g} / \mathrm{cm}^{2}\right.$; Collagen Reagent Horm, Hormon Chemie, Munich, FRG) as model of injured vessel wall (17).

Perfusions were carried out as has been described previously (16). In short: The perfusion chambers were rinsed with prewarmed $\left(37^{\circ} \mathrm{C}\right)$ Hepes-buffered saline (HBS: Hepes $10 \mathrm{mM}, \mathrm{NaCl} 0.15 \mathrm{M}, \mathrm{pH} 7.4$ ). Perfusates (volume $14 \mathrm{ml}$ ) prewarmed for $15 \mathrm{~min}$ at $37^{\circ} \mathrm{C}$, were then recirculated for $5 \mathrm{~min}$ at various shear rates $(300 / \mathrm{s}$, to approach lower "venous" shear rates, or $1,300 / \mathrm{s}$, as a shear rate typical for the "arterial" vascular bed). After each perfusion run the arteries and coverslips were removed and the system rinsed thoroughly with HBS. The coverslips and vessel wall segments were fixed with respectively $0.5 \%$ and $2.5 \%$ glutardialdehyde in PBS ( $1: 10 \mathrm{vol} / \mathrm{vol} 0.1 \mathrm{M}$ sodiumphosphate buffer, pH 7.4 in $150 \mathrm{mM} \mathrm{NaCl}$ ), followed by osmium tetroxide (2\%) postfixation, ethanol dehydration, and subsequent embedding in epon (12). Thermanox coverslips were removed from the embedded collagen surface by thermoshock.

Perfusion studies with LMWH-anticoagulated blood. Except for the method of anticoagulation and the perfused surface, perfusions were carried out as described above. Blood for perfusion experiments was collected into 1 / 10 vol of LMWH (Fragmin, Kabi Vitrum, Stockholm, Sweden ) 200 anti-Xa U/ml saline. The LMWH concentration was subsequently adjusted to a plasma concentration of $33 \mathrm{U} / \mathrm{ml}$ in both patients and controls. Experiments (not shown) in which equal 
amount of LMWH were added to patient or to control plasma (both anticoagulated with $20 \mathrm{mM}$ trisodium citrate) showed comparable anti-Xa activity in both plasmas. Perfusates were standardized for hematocrit and platelets as described in the citrate anticoagulated perfusions.

As a perfusion surface we used matrix from human umbilical vein endothelial cells (HUVECs). HUVECs were isolated from umbilical cords according to the method originally described by Jaffe et al. (23) with some minor modifications (24). HUVECs of the second passage were identified by their typical characteristics and subcultured on Thermanox coverslips that were previously coated and glutardialdehyde fixed with gelatin (24). Cell monolayers were grown to confluence $\left(50,000\right.$ cells $\left./ \mathrm{cm}^{2}\right)$ in 5-7 d. HUVECs' tissue factor deposition in their ECM, was stimulated by addition of PMA (Sigma Chemical Co.) to the culture medium (16). PMA was dissolved in DMSO and subsequently diluted 1:1,000 vol/ $\mathrm{vol}$ in the culture medium: $20 \mathrm{ng}$ $\mathrm{PMA} / \mathrm{ml}$. Addition of DMSO alone in the same concentration had no influence on the cells. After $10 \mathrm{~h}$, matrix of the stimulated HUVEC was isolated by $0.1 \mathrm{M} \mathrm{NH}_{4} \mathrm{OH}$. Coverslips with isolated ECM were washed three times with PBS and used for perfusion studies. The perfused coverslips were fixed, dehydrated, epon embedded, and "thermoshocked" as described above. For microphotographs of en face morphology, coverslips were stained after perfusion and fixation, with May-Grunwald/Giemsa.

Evaluation of platelet deposition and thrombus formation. Sections of the epon-embedded collagen, $1 \mu \mathrm{m}$ thick, perfused matrix, and vessel wall segments were prepared and stained with methylene blue and basic fuchsin for evaluation of platelet deposition and aggregation. Platelet deposition and aggregation were evaluated by light microscopy (Dialux $20 \mathrm{~EB}$, Leitz GmbH, Wetzlar, FRG) at 1,000 magnification, using a specially constructed eyepiece micrometer in the ocular. At least 1,000 intersection points at $10-\mu \mathrm{m}$ intervals, evenly distributed over the total of the perfused surfaces were evaluated for each section. Platelet adherence was determined from the percentage of artery, ECM of collagen surface directly covered by contact platelets, platelets spread out on the surface and platelet aggregates (between 2 and $10 \mu \mathrm{m}$ in height), according to the criteria described by Baumgartner et al. (25). Aggregate formation was defined and expressed as the percentage of spread platelets covered with aggregated platelets and was therefore a measure of platelet-platelet interaction independent of total surface coverage. Microscopically visible fibrin deposited on perfused ECM and above $2 \mu \mathrm{m}$ in height was expressed as percentage surface coverage.

Studies with reconstituted perfusates. Additional perfusions with reconstituted blood were performed to exclude the possibility of an adhesion defect in nephrotic platelets that would conceal adhesion promoting qualities of nephrotic plasma. Plasma or washed platelets of four patients of group 2 were respectively mixed with plasma or washed platelets and red blood cells from control subjects. The patients had high levels of vWF-Ag $(2.11 \pm 0.43 \mathrm{U} / \mathrm{ml})$ and fibrinogen $(6.7 \pm 1.3 \mathrm{~g} /$ liter). Perfusions were performed in duplicate for $5 \mathrm{~min}$ at a shear of $1,300 \mathrm{~s}^{-1}$ over inverted umbilical artery segments. The artery segments were evaluated as described above.

Studies with varying fibrinogen levels. The effect of increased fibrinogen levels, such as occur in the nephrotic syndrome, on plateletvessel wall interaction and in vitro platelet aggregation was evaluated by adding fibrinogen to blood of normal donors. This approach was technically more feasible than defibrinating blood of nephrotic patients. In the later procedure it is hard to avoid substantial loss of vWF with the defibrination. Purified fibrinogen (Kabi Vitrum; dissolved to $400 \mathrm{mg} / \mathrm{ml}$ in and dialyzed for $6 \mathrm{~h}$ against $0.15 \mathrm{M} \mathrm{NaCl}$ was added to blood of control subjects until concentrations of 2,3 , and 4 or 4.5 $\mathrm{g} /$ liter were obtained. Because purified fibrinogen contained high levels of free FPA, we could not measure the matrix-induced increase in FPA in perfusates with added fibrinogen. Human albumin in $0.15 \mathrm{M}$ $\mathrm{NaCl}$ in the same concentrations was added to perfusates to serve as control. Platelet aggregation studies after stimulation with ADP, AA, and spontaneous aggregation were also performed with control PRP to which fibrinogen was added in similar fashion.
Analytical methods. EDTA ( $2 \mathrm{mM}$ ) anticoagulated whole blood was collected for determination of hematocrit, mean cell volume, and platelet count (model-S. Coulter, Harpenden, UK). Fibrinogen, factors II, V, VII, and X, antithrombin III (AT III), and vWF-related properties were determined in blood collected into 0.1 vol $130 \mathrm{mM}$ trisodium citrate. Reference plasma was a freshly frozen plasma pool from 40 normal subjects, stored at $-70^{\circ} \mathrm{C}$. Plasma fibrinogen level was determined according to Clauss (26). Factor II, V, VII, and X coagulant activity was measured in an one-stage clotting assay with commercially available deficient plasmas (George King Bio-Medical Inc., Overland Park, KS) as substrate. AT III was assayed with an AT III-kit (Kabi-Vitrum) according to the manufacturers instructions. vWF-related antigen was determined by an ELISA (27), the ristocetin cofactor activity was measured with formalin-fixed platelets and $1 \mathrm{mg} / \mathrm{ml}$ ristocetin sulfate (H. Lundbeck \& Co., Copenhagen, Denmark) (28). Albumin, creatinine, and proteinuria were determined by standard laboratory techniques.

Measurements in the perfusates. A radioimmuno-assay kit (Mallinckrodt Inc., St. Louis, MO) was used for FPA measurements. A enzyme immunoassay (Enzygnost F1 + 2, Behring, Marburg, FRG) was used to determine the activation peptide of prothrombin when thrombin is formed. Before, during, and after perfusion, samples ( 900 $\mu \mathrm{l})$ from the perfusate were collected and added to $100 \mu \mathrm{l}$ of anticoagulant mixture provided by the FPA-kit. FPA and fragment $1+2$ generation was calculated in these samples by subtracting baseline concentrations from concentrations in the perfusate at the end of the perfusions. FPA generation was expressed in nanograms per milliliter plasma, fragment $1+2$ in nanomolar.

Statistical analysis. Data are expressed as mean \pm SD. The significance of differences between patient and control data obtained in various experiments were compared with the Student's $t$ test for paired data. Linear regression was applied to test for correlations between fibrinogen levels and platelet-vessel wall interaction during the various experiments. Differences in the fibrinogen studies were tested with ANOVA with shear rate, stimulus concentration and fibrinogen concentration as independent variables. The differences between the means were analyzed at $1 \%$ and $5 \%$ significance level by the least significance difference test.

\section{Results}

Aggregation studies within suspension. Non aggregated (single) platelets remaining in PRP after stimulation with ADP as well as after stimulation with AA (Table II) were significantly decreased in PRP of nephrotic subjects (group 1). This increased aggregability in nephrotic PRP was particularly apparent at low concentrations of the stimulating agent, when control subjects displayed almost no platelet aggregation. In addition, we found significant spontaneous aggregation in the nephrotic PRP after mere stirring.

Platelet adhesion and aggregate formation with flowing citrated blood. Except for increased levels of fibrinogen, nephrotic subjects of group $1(n=10)$ also had significantly increased levels of vWF-antigen and vWF-ristocetin cofactor activity. In addition, red blood cell deformability was reduced (Table IIIB). These abnormalities-known as platelet adhesion promoting factors-were present in all the patients. Platelet adhesion (expressed as percentage surface area covered with platelets) to deendothelialized umbilical artery, however, did not differ between nephrotic patients (group 1) and control subjects (Table IIIA). Spread platelets were subdivided in spread platelets without and with aggregates $>2 \mu \mathrm{m}$ on top. Platelet aggregate formation, thus defined and expressed as percentage of total platelet coverage was slightly but significantly higher in the nephrotic subjects (Table III). Perfusions over 
Table II. Platelet Aggregation Studies in Suspension after Stimulation with ADP and $A A$ and Spontaneous Aggregation (Patient Group 1)

\begin{tabular}{lcc}
\hline & Patients $(n=10)$ & Controls $(n=10)$ \\
\hline ADP-stimulated aggregation $(\mu M)$ & & \\
5 & $19 \pm 8$ & $24 \pm 16$ \\
2.5 & $35 \pm 14^{*}$ & $52 \pm 12$ \\
1.67 & $41 \pm 9^{*}$ & $60 \pm 14$ \\
0.84 & $52 \pm 9^{\ddagger}$ & $79 \pm 9$ \\
AA $(m M)$ & & \\
1.5 & $14 \pm 12$ & $36 \pm 26$ \\
0.75 & $26 \pm 19$ & $55 \pm 33$ \\
0.375 & $27 \pm 18^{*}$ & $82 \pm 6$ \\
0.250 & $39 \pm 23^{*}$ & $89 \pm 8$ \\
Spontaneous aggregation & $69.1 \pm 20.9^{*}$ & $86.9 \pm 10.2$ \\
\hline
\end{tabular}

Platelet aggregation is expressed as percentage single (nonaggregated) platelets of initial platelet counts in PRP after 3-min stimulation with ADP and AA are given at 3-min stimulation or after 15-min mere stirring. All values expressed as means \pm SD. ${ }^{*} P<0.05,{ }^{\ddagger} P$ $<0.01$ compared to control subjects.

umbilical arteries performed at lower $(300 / \mathrm{s})$ shear rates as model for venous flow resulted in an expected lowered platelet adherence. In our study these perfusions at lower shear did not enhance the difference in aggregate formation between nephrotics and control subjects. Platelet adhesion to sprayed collagen at $1,300 / \mathrm{s}$ shear was less in both patients and control subjects than to umbilical arteries (Table IIIA). However, ag-

Table III. Platelet Adhesion and Aggregate Formation at Umbilical Arteries and Sprayed Collagen $(A)$ Related to Factors Determining Platelet-Vessel Wall Interaction (B, Patient Group 1)

\begin{tabular}{lcc}
\hline \multicolumn{1}{c}{ A } & Patients $(n=10)$ & Controls $(n=10)$ \\
\hline Umbilical arteries (shear 1,300/s) & & \\
Adhesion (\%) & $51.9 \pm 16.1$ & $55.3 \pm 14.6$ \\
Aggregate (\%) & $23.7 \pm 6.1^{*}$ & $20.8 \pm 6.6$ \\
Umbilical arteries (shear 300/s) & & \\
Adhesion (\%) & $38.7 \pm 5.4$ & $44.5 \pm 5.5$ \\
Aggregate (\%) & $22.9 \pm 3.2^{*}$ & $18.8 \pm 6.2$ \\
Collagen (shear 1300/s) & & \\
Adhesion (\%) & $33 \pm 7.5$ & $35.9 \pm 9.7$ \\
Aggregate (\%) & $64.0 \pm 11.6^{*}$ & $58.5 \pm 12.9$ \\
B & & \\
Deformability index & $0.21 \pm 0.08^{*}$ & $0.37 \pm 0.02$ \\
Fibrinogen $(\mathrm{g} / \mathrm{liter})$ & $7.2 \pm 2.0^{\ddagger}$ & $2.8 \pm 0.5$ \\
vWF-ag $(U / \mathrm{ml})$ & $2.18 \pm 0.55^{\ddagger}$ & $0.73 \pm 0.08$ \\
vWF-RCof $(U / \mathrm{ml})$ & $1.59 \pm 0.39^{\ddagger}$ & $0.65 \pm 0.05$ \\
& & \\
\hline
\end{tabular}

$\mathrm{RBC}$ deformability index is determined at $30 \mathrm{~N} / \mathrm{m}^{2}$. Abbreviations: von Willebrand antigen (vWF-Ag) and ristocetin cofactor (vWFRCof). Aggregate formation is expressed as the percentage of total platelet adhesion consisting of aggregate formation $(>2 \mu \mathrm{m})$. All values are given as mean \pm SD. ${ }^{*} P<0.05,{ }^{\ddagger} P<0.01$ compared to control subjects.

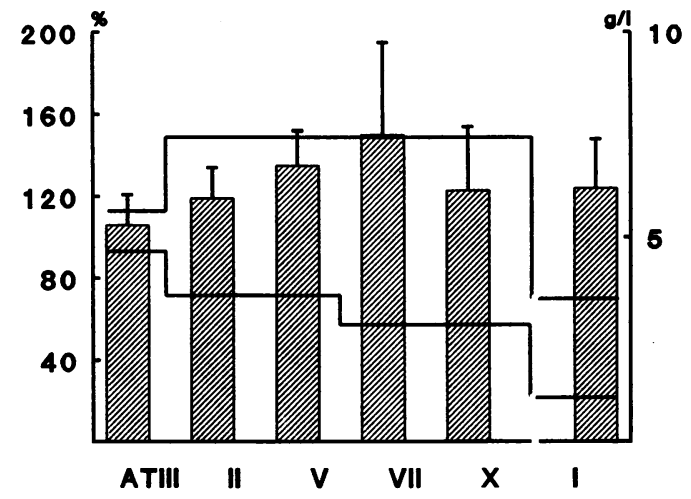

Figure 1. Means \pm SD of clotting factors (II, V, VII, X, I) and AT III of patient group 2. Values are expressed as percentage (ATIII, II, V, VII, X), or as $\mathrm{g} /$ liter (factor I (=fibrinogen). The solid lines refer to the reference range (mean $\pm 2 S D$ ).

gregate formation was increased in both patients and control subjects. Again, platelet adhesion on collagen between nephrotics and control subjects did not differ, whereas aggregate formation was slightly increased in the nephrotic patients. Platelet counts at the end of the perfusions were always $>100,000 / \mu \mathrm{l}$. Above this value, platelet adhesion is not determined by varying platelet counts (16).

Platelet thrombi and fibrin deposition by matrix-induced thrombin formation. Levels of clotting factors and antithrombin-III were measured in the nephrotic patients of group 2 . Except for a large increase in fibrinogen all were within or moderately above (factor VII) the normal range (Fig. 1). In perfusions with LMWH-anticoagulated blood of these patients over endothelial cell ECM, platelet adhesion was decreased as compared to normal controls (Table IVA). Aggregate formation on adherent platelets was also reduced compared to controls. In the patient perfusions, however, there was a more than twofold increase in fibrin deposition directly on the ECM ( Table IVA, Fig. 2). Additionally, FPA generation, as more quan-

Table IV. (A) Platelet Adhesion, Aggregate Formation, and Fibrin Deposition on Tissue Factor-rich Endothelial Cell Matrix ECM; (B) Fragment $1+2$ and FPA Levels before and Generation during Perfusions with LMWH-Anticoagulated Blood from Nephrotic Subjects (Group 2) and Control

\begin{tabular}{llcc}
\hline \multicolumn{1}{c}{ ECM (shear 1300/s) } & Patients $(n=10)$ & Controls $(n=10)$ \\
\hline A & Adhesion (\%) & $24.1 \pm 5.6^{*}$ & $41.8 \pm 5.5$ \\
& Aggregate (\%) & $48.3 \pm 5.1^{*}$ & $57.5 \pm 2.2$ \\
& Fibrin deposition (\%) & $60.0 \pm 17.3^{\ddagger}$ & $25.5 \pm 1.8$ \\
& & & \\
B & F1 + 2 preperfusion $(\mathrm{ng} / \mathrm{ml})$ & $3.6 \pm 1.7$ & $1.8 \pm 0.9$ \\
& F1 + 2 generation $(\mathrm{ng} / \mathrm{ml})$ & $5.4 \pm 2.9$ & $3.4 \pm 4.0$ \\
& FPA preperfusion $(\mathrm{ng} / \mathrm{ml})$ & $24.9 \pm 11.3^{\ddagger}$ & $7.8 \pm 2.8$ \\
& FPA generation $(n g / m l)$ & $303 \pm 187^{\ddagger}$ & $107 \pm 76$ \\
& Fibrinogen $(\mathrm{g} / \mathrm{liter})$ & $6.2 \pm 1.9^{\ddagger}$ & $2.9 \pm 0.6$
\end{tabular}

Adhesion is expressed as percentage surface coverage. Aggregate formation is expressed as the percentage of the platelet adhesion consisting of aggregate formation ( $>2 \mu \mathrm{m})$. Fibrin deposition $(>2 \mu \mathrm{m})$ expressed as percentage of total surface. All values are given as mean \pm SD. ${ }^{*} P<0.05 ;^{\ddagger} P<0.01$ compared to controls. 


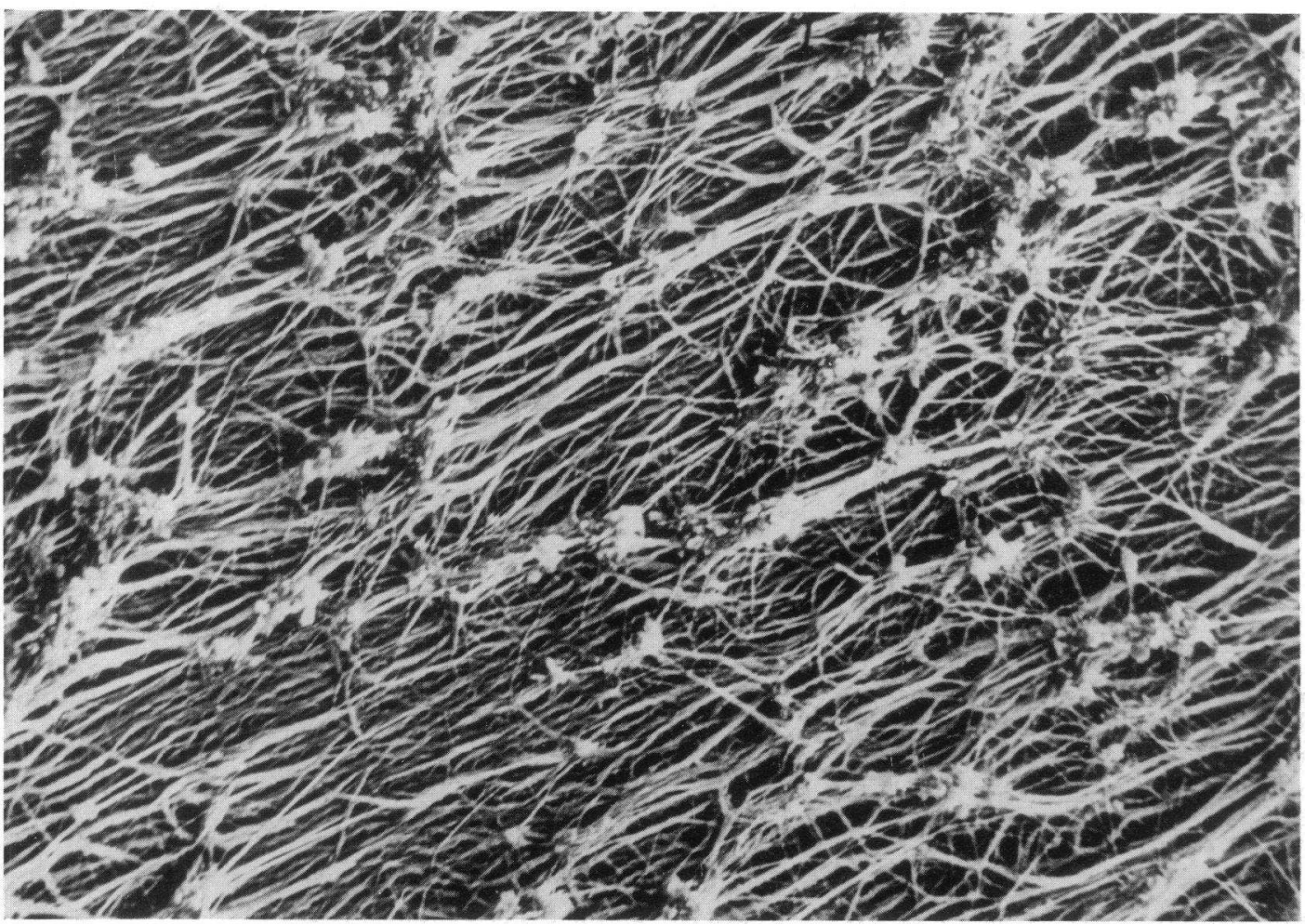

Figure 2. Scanning electron micrograph of fibrin depositions on tissue-factor rich endothelial cell matrix in a nephrotic subject, after 5-min perfusion with whole blood anticoagulated with LMWH. titative measure of the fibrinogen conversion to fibrin, was threefold higher than in controls (Table IVB). To correlate the level of hyperfibrinogenemia in patients ( Table IVB) with FPA generation and fibrin deposition we expressed patient data from all experiments as percentage of values obtained in parallel performed experiments with control blood. Thus expressed, thrombin-induced FPA generation in patients correlated strongly with the substrate (fibrinogen) concentration (Fig. 3; $r$ $=0.81, P<0.01)$. Initial fibrinogen levels also correlated with fibrin deposition ( $r=0.75, P<0.05)$. The increased levels of FPA in patient blood before perfusions (baseline) possibly reflect increased fibrin generation in vivo or at blood collection (Table IVB). Matrix-induced thrombin generation in perfusates, as reflected by the fragment $1+2$ generation in the perfusate, was not different between patients and controls ( Table IVB).

Studies with reconstituted perfusates. Perfusion of platelets of control subjects reconstituted in nephrotic plasma over inverted umbilical arteries did not demonstrate increased platelet adhesion when compared to perfusions with control platelets reconstituted in control plasma: $58.2 \pm 5.4 \%$ vs. $54.1 \pm 4.6 \%$ of surface coverage. Nor did control platelets in nephrotic plasma show increased aggregate formation $(>2 \mu \mathrm{m})$ compared to control platelets in control plasma: $47.4 \pm 5.8 \%$ vs. $49.5 \pm 5.5 \%$ of total platelet adhesion. Similarly, there was no significant difference in adhesion and aggregate formation whether nephrotic platelets were reconstituted in their own plasma or control plasma; platelet adhesion was respectively $59.1 \pm 6.6 \%$ (nephrotic plasma) vs. $55.8 \pm 5.1 \%$ (control plasma) and aggregate formation respectively $36.9 \pm 7.8 \%$ vs. $34.8 \pm 6.3 \%$. However, aggregate formation was lower $(P<0.05)$ in the reconstituted perfusion studies with nephrotic platelets compared to those with control platelets.
Fibrinogen studies. As main interlinking protein of activated platelets and as substrate of thrombin-induced fibrin generation, we investigated whether high levels of fibrinogen in normal blood could would have similar effects as were obtained in the experiments with nephrotic patients. For this purpose, the fibrinogen concentration in control blood was increased. Hyperfibrinogenemia stimulated in vitro platelet aggregation (Table V). Addition of fibrinogen to the perfusate resulted in a progressive decrease in platelet adhesion to umbili-

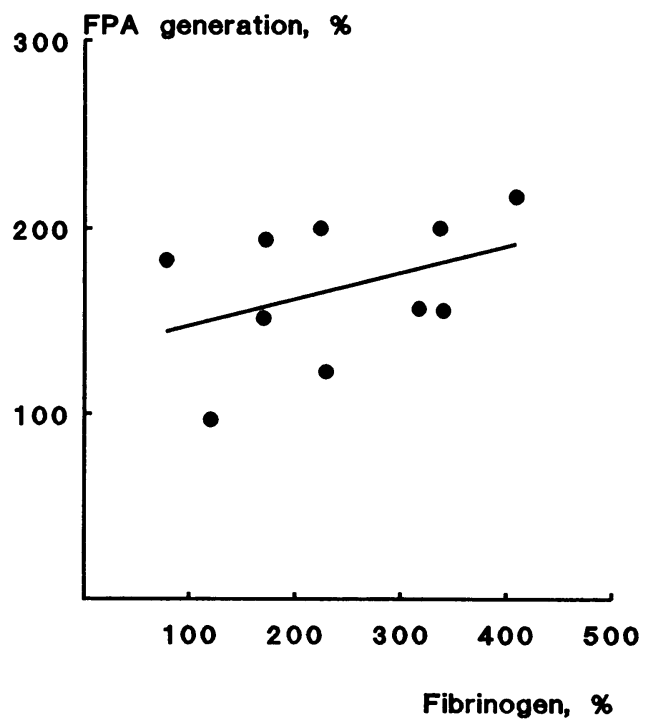

Figure 3. FPA generation versus baseline fibrinogen in patient group 2 , both expressed as percentage of parallel performed control experiment. Regression line, $r=0.81, P<0.01$. 
Table V. Nonaggregated Platelets Remaining in PRP of Control Subjects after Stimulation with ADP, AA, and Mere Stirring; Aggregability as Function of Different Fibrinogen Concentrations in PRP

\begin{tabular}{lllc}
\hline \multicolumn{1}{c}{ Fibrinogen concentration } & \multicolumn{1}{c}{2.0} & 3.0 & 4.5 \\
\hline \multicolumn{1}{c}{$(g /$ liter $)$} & & & \\
ADP-stimulated aggregation $(\mu M)$ & & & \\
5 & $34 \pm 12$ & $27 \pm 14$ & $22 \pm 11^{*}$ \\
2.5 & $62 \pm 9$ & $47 \pm 10^{*}$ & $36 \pm 14^{\ddagger}$ \\
AA $(m M)$ & & & \\
$\quad 1.5$ & $8 \pm 2$ & $7 \pm 3$ & $5 \pm 3$ \\
0.75 & $93 \pm 6$ & $90 \pm 7$ & $73 \pm 9^{*}$ \\
Spontaneous aggregation & $90 \pm 8$ & $90 \pm 7$ & $77 \pm 7^{*}$ \\
& & & \\
\hline
\end{tabular}

Higher platelet aggregability is expressed in a lower percentage (of initial platelet counts in PRP) single (nonaggregated) platelets remaining in PRP after 3-min stimulation with ADP and AA or after 15 -min mere stirring. Values are mean $\pm \mathrm{SD}$ of duplicate experiments. ${ }^{*} P<0.05,{ }^{\ddagger} P<0.01$, compared to values obtained with PRP with $2.0 \mathrm{~g} /$ liter fibrinogen.

cal arteries (Table VI). This was particularly apparent at high shear rates at which platelet adhesion is normally increased. Aggregate formation also decreased with increasing fibrinogen concentrations both at $1,300 / \mathrm{s}$ and $300 / \mathrm{s}$ shear. Similarly, platelet adhesion and aggregate formation decreased at increasing fibrinogen concentrations in LMWH perfusions over ECM. Most of the perfusion surface in these perfusions was covered with fibrin both at high and low fibrinogen concentrations. However, at the high fibrinogen concentration larger fibrin depositions had formed (Table VII).

\section{Discussion}

Increased platelet adhesion, aggregate formation, and coagulation at injured vessel wall have been proposed as important

Table VI. Platelet Adhesion and Aggregate Formation on Umbilical Arteries in Control Perfusates at Different Concentrations of Fibrinogen

\begin{tabular}{ccc}
\hline Fibrinogen concn. & Adhesion & Aggregate \\
\hline (g/liter) & & \\
Shear rate $1,300 / \mathrm{s}$ & & \\
2.0 & $59.3 \pm 7.4$ & $35.5 \pm 10.8$ \\
3.0 & $43.3 \pm 5.7^{*}$ & $22.2 \pm 7.0^{*}$ \\
4.5 & $35.6 \pm 5.6^{\ddagger}$ & $19.3 \pm 6.3^{*}$ \\
Shear rate 300/s & & \\
2.0 & $44.0 \pm 6.7$ & $25.4 \pm 6.5$ \\
3.0 & $35.9 \pm 6.5^{*}$ & $19.7 \pm 8.9^{*}$ \\
4.5 & $34.9 \pm 2.9^{*}$ & $12.6 \pm 3.9^{\ddagger}$ \\
\hline
\end{tabular}

Adhesion is expressed as percentage surface coverage. Aggregate formation is expressed as the percentage of the platelet adhesion consisting of aggregate formation $(>2 \mu \mathrm{m})$. Values are given as mean \pm SD of duplicate experiments. ${ }^{*} P<0.05$; ${ }^{\ddagger} P<0.01$ compared to low fibrinogen $(2.0 \mathrm{~g} /$ liter $)$ concentration.
Table VII. Platelet Adhesion, Aggregate Formation, and Fibrin Depositions on ECM at Different Fibrinogen Concentrations in Control Perfusate

\begin{tabular}{ccccc}
\hline Fibrinogen & Adhesion & Aggregate & Fibrin $(>2,>5 \mu \mathrm{m})$ & Fibrin $(>5 \mu \mathrm{m})$ \\
\hline g/liter & & \multicolumn{3}{c}{} \\
2.0 & $26 \pm 5$ & $72 \pm 19$ & $63 \pm 38$ & $10 \pm 6$ \\
4.0 & $21 \pm 5$ & $57 \pm 13^{*}$ & $31 \pm 20^{*}$ & $39 \pm 21^{*}$
\end{tabular}

Adhesion is expressed as percentage surface coverage. AG/TS, aggregate formation ( $>2 \mu \mathrm{m})$ (AG) expressed as percentage of total surface coverage (TS). Fibrin deposition ( $>2 \mu \mathrm{m}$ ) expressed as percentage of total surface. Values are given as mean $\pm \mathrm{SD}$ of duplicate experiments. ${ }^{*} P<0.05$; compared to low fibrinogen concentration.

mechanisms in the development of thrombosis (29) and atherosclerosis ( 3 ). It has been postulated that increased plateletvessel wall, platelet-platelet interaction, and hypercoagulability are also important features of the prethrombotic state in patients with nephrotic syndrome $(1,2)$. This notion has been extrapolated from in vitro observations, showing abnormalities in platelet function and coagulation factors (5-11). Although flow is a crucial determinant of both platelet- and coagulationdependent hemostasis, no information is available on the significance of these abnormalities under flow conditions in the nephrotic syndrome.

The present study confirms that platelet aggregability in suspension is increased after stimulation with ADP, arachidonic acid and by mere stirring ( Table II). Platelet hyperaggregability in the nephrotic syndrome probably has a multifactorial genesis and has been associated with low serum albumin, hypercholesterolemia, and hyperfibrinogenemia $(8,9)$. Only few studies deal with the mechanism and relevance of these associated changes. Platelet function after stimulation with AA normalizes after correction of hypoalbuminemia in vitro and in vivo $(9,10)$. Hypoalbuminemia might increase cyclooxygenase's efficiency to form the proaggregatory thromboxane $A_{2}$ in the presence of a higher concentration of free arachidonic acid that is normally bound to albumin $(8,9)$. However, platelet hyperaggregability to other stimuli such as ADP, collagen, and ristocetin could not be related to hypoalbuminemia (8, 11). Fibrinogen links GPIIbIIIa receptors on activated platelets (30). Since hyperfibrinogenemia is characteristic of the nephrotic syndrome, it seems logical to postulate a role for fibrinogen in the platelet dysfunction in nephrosis. The present observation that platelet aggregation in suspension increases with increasing concentrations of fibrinogen supports this hypothesis. Meade et al. ( 31 ) reported increased aggregation velocity at higher fibrinogen concentrations; the maximum aggregation response, however, decreased with increasing fibrinogen concentrations. The latter difference might be explained by the fact that in our study single (nonaggregated) platelets were measured instead of light transmission which is dependent on the final size of the aggregates.

Increased platelet aggregation tests in suspension are frequently used as indicator for increased platelet-vessel wall interaction in vivo (i.e., under flow conditions) $(1,2)$. Moreover, vWF was increased and red blood cell deformability decreased in our patients ( Table IIIB). These are conditions respectively associated with increased platelet adhesion, and more effective platelet transport to the vessel wall (13-15). However, during 
perfusions with citrated blood we found a normal platelet adhesion and only a modestly increased platelet aggregation ( $\mathrm{Ta}$ ble IIIA). The perfusion system with umbilical arteries is well capable of platelet adhesion above the $50 \%$ found in our study (22). Therefore, the absence of increased platelet adhesion in nephrotic subjects could not be explained by a near maximal platelet adhesion stimulus. Moreover, perfusion studies over tissue factor rich endothelial cell matrix, in which blood-anticoagulation with LMWH allowed matrix initiated thrombin formation, showed even less platelet adhesion and aggregate formation in nephrotic subjects than in controls. The decrease in platelet adherence in this model was associated with extensive fibrin depositions on the matrix (ECM) (Table IVA). Therefore, the expected increase in platelet-vessel wall interaction in flowing nephrotic blood was in some way counteracted while fibrin deposition was increased.

To exclude the possibility that a platelet defect in the nephrotic syndrome accounted for the absence of increased platelet-vessel wall interaction, we also perfused umbilical arteries with reconstituted blood, i.e., washed platelets of control subjects in control plasma and nephrotic plasma, and washed platelets of nephrotic subjects in nephrotic plasma and control plasma. Similar to the whole blood perfusion studies nephrotic plasma did not increase platelet adhesion compared to control plasma, neither with control platelets nor with nephrotic platelets. There was a decreased aggregate formation in the reconstitution perfusions with nephrotic platelets. However, this was the case both in nephrotic plasma as well as in control plasma. Since nephrotic platelets are hyperaggregable in nephrotic plasma (in aggregometer studies and in lesser degree in whole blood perfusion studies), this phenomenon is likely to be due to loss of hyperaggregable platelets (and thus selection of relatively refractory platelets) during the centrifugation and washing procedures which are necessary for the isolation of platelets. The presence of a platelet adhesion defect therefore seems not a satisfactory explanation for the absence of an increased platelet-vessel wall interaction.

To evaluate the isolated role of hyperfibrinogenemia apart from other factors, we performed additional experiments with control blood in which the fibrinogen concentration was increased. In these studies, addition of fibrinogen to control blood could mimic most of the observations in nephrotic subjects. Fibrinogen again promoted platelet aggregation in suspension, but it did not stimulate platelet-vessel wall interaction under flow conditions. In fact, perfusion studies with control blood at increasing fibrinogen concentrations revealed a decrease in both platelet adhesion and aggregate formation; in LMWH perfusions decreased platelet adherence was replaced by more pronounced fibrin deposition. In as far as this observation can be extrapolated to our nephrotic patients, hyperfibrinogenemia in patients may thus counteract vWF and red blood cell enhanced platelet adhesion.

The mechanism of these phenomena is not clear yet. One possible explanation might be that at low shear stress, as in the aggregometer, fibrinogen-induced platelet aggregates are not exposed to strong dissolving forces. Instead, in the perfusion system where much higher shear stress exist these aggregates might be dissolved. Alternatively, aggregation in the perfusate was increased, as in the in vitro aggregation tests, so that less platelets were available for adhesion. In agreement with this notion several studies have reported in vivo increased levels of the platelet release product $\beta$-thromboglobulin $(32,33)$. How- ever, we previously demonstrated that platelet availability is not determining platelet adhesion in the perfusion system as long as the platelet count in the perfusate is more than $100,000 / \mu 1$, as was the case in the present study (16). Fibrin depositions bind to subendothelium and might thus interfere with ligand systems in the subendothelium, thereby decreasing platelet adhesion. The present observation that platelet adhesion decreases with increasing fibrin depositions supports this notion. Finally, it is tempting to speculate that fibrinogen or fibrin interfered with vWF-GPIIbIIIa mediated adhesive processes. Support for this hypothesis was derived from results in perfusions over laminin surfaces on which platelets adhere via the VLA-6 integrin receptor supported by vWF-GPIb interaction and independent of vWF-GPIIbIIIa interactions. Contrary to the present perfusions, adhesion to this laminin was not decreased by adding extra fibrinogen to the perfusate (unpublished observations, S. Endenburg, Utrecht ).

The increased fibrin deposition at tissue factor rich matrix was accompanied by a threefold increase in FPA generation in nephrotic perfusates above control values. These findings reflect thrombin-induced fibrin generation and might be caused by increased thrombin formation, by less effective thrombin inhibition or by more effective fibrinogen-fibrin transition. Generation of fragment $1+2$, as a measure of activation of prothrombin into thrombin, was not different from control. This suggests that the enhanced fibrin formation is not determined by increased thrombin formation. This might implicate that thrombin is longer active at matrix perfused with nephrotic blood. One cause of prolonged action of thrombin might be a reduction in levels of AT III. Although depressed levels of AT III have been reported frequently in patients with heavy proteinuria (7), plasma levels were normal in the present study (Fig. 1). In addition, depressed levels of AT III would be associated with increased formation of thrombin, which was not apparent from the (normal) fragment $1+2$ formation in our study. Alternatively, one might postulate that up to the supraphysiological plasma levels found in our patients, fibrinogen availability as a substrate is rate limiting for tissue factordependent thrombin in inducing fibrin formation. This possibility is suggested by the strong correlation between initial fibrinogen level and FPA generation in the nephrotic subjects (Fig. 3). Further support comes from in vivo observations in rabbits where intravascular fibrin deposition increased with elevation of the plasma fibrinogen level (34). Finally, although perfusion time was only $5 \mathrm{~min}$, we cannot exclude that decreased or less effective fibrinolysis in nephrosis also may have contributed somewhat to the extensiveness of the depositions.

Extrapolated from our experiments, the high risk for thromboembolic complications in nephrotic subjects may depend on fibrin depositions, even at relatively high shear rates. In vivo fibrin deposition in nephrotic subjects is also suggested by elevated baseline FPA levels in the present study (Table IVB), as well as in studies by others (35). Fibrin deposition normally increases with lower shear rates and promotes inclusion of RBCs and other cell elements (12). An increased tendency to form these "mixed-thrombi" will therefore be most obvious at lower shears in the venous vasculature or at sites of turbulence, such as atherosclerotic lesions. Furthermore, fibrin depositions are also present in atherosclerotic lesions, and are proposed to contribute to atherosclerosis by causing endothelial cell disorganisation, by stimulating the proliferation of vascular smooth muscle cells and by providing an adsorptive sur- 
face for LDL accumulation (36, 37). Finally, in nephrotic patients elevated fibrinogen in renal vein blood correlated with intraglomerular fibrin depositions, and may contribute to the development of glomerular injury (38).

In summary, the present studies challenge the concept of increased in vivo platelet-vessel wall interaction in the nephrotic syndrome. We suggest that a plasma factor, presumably fibrinogen, counteracts this interaction. Therefore, in vitro aggregation tests do not predict platelet function under flow conditions in the nephrotic syndrome. Our observations also suggest that the thrombotic risk in nephrosis is determined by coagulation abnormalities rather than by platelet dysfunction. Hyperfibrinogenemia may be the major factor determining the thrombotic risk in nephrosis by inducing fibrin depositions proportional to the fibrinogen level. This also implies that therapy for hypercoagulability in the nephrotic syndrome should focus on this abnormality.

\section{Acknowledgments}

This study was supported by the Dutch Kidney Foundation. A.J. Rabelink is a fellow of the Royal Dutch Academy of Sciences (KNAW).

\section{References}

1. Cameron, J. S. 1984. Coagulation and thromboembolic complications in the nephrotic syndrome. Adv. Nephrol. 75-114.

2. Llach, F. 1985. Hypercoaguability, renal vein thrombosis and other thrombotic complications of nephrotic syndrome. Kidney Int. 28:429-439.

3. Ross, R. 1986. The pathogenesis of atherosclerosis: an update. N. Engl. J. Med. 314:488-500.

4. Barnes, J. L., and M. A. Ventakatachalam. 1985. The role of platelets and polycationic mediators in glomerular vascular injury. Semin. Nephrol. 5:57-68.

5. Thompson, C., C. D. Forbes, C. R. M. Prentice, and A. C. Kennedy. 1974. Changes in blood coagulation and fibrinolysis in the nephrotic syndrome. $Q$. $J$. Med. 43:399-407.

6. Kendall, A. G., R. E. Lohmann, and J. B. Dossetor. 1971. Nephrotic syndrome: a hypercoaguable state. Arch. Intern. Med. 127:1021-1027.

7. Kauffman, R. H., J. J. Veltkamp, N. H. van Tilburg, and L. A. van Es. 1978. Acquired antithrombin III deficiency and thrombosis in the nephrotic syndrome. Am. J. Med. 65:607-613.

8. Machleidt, C., T. Mettang, E. Starz, J. Weber, T. Risler, and U. Kuhlmann. 1989. Multifactorial genesis of enhanced platelet aggregability in patients with nephrotic syndrome. Kidney Int. 36:1119-1124.

9. Remuzzi, G., G. Mecca, D. Marchesi, M. Livio, G. De Gaetano, M. B. Donati, and M. J. Siver. 1979. Platelet hyperaggregability and the nephrotic syndrome. Thromb. Res. 16:345-354.

10. Jackson, C. A., M. Creaves, A. D. Patterson, C. B. Brown, and F. E. Preston. 1982. Relationship between platelet aggregation, thromboxane synthesis and albumin concentration in nephrotic syndrome. Br. J. Haematol. 52:69-75.

11. Bennet, A., and J. S. Cameron. 1987. Platelet hyperaggregability in the nephrotic syndrome which is not dependent on arachidonic acid metabolism or on albumin concentration. Clin. Nephrol. 27:182-188.

12. Baumgartner, H. R. 1973. The role of blood flow in platelet adhesion, fibrin deposition and formation of mural thrombi. Microvasc. Res. 5:167-179.

13. Turitto, V. T., H. J. Weiss, and H. R. Baumgartner. 1979. Rheologica factors influencing platelet interaction with vessel surfaces. $J$. Rheol. 23:735-749.

14. Aarts, P. A. M. M., R. H. Heethaar, and J. J. Sixma. 1984. Red blood cell deformability influences platelets-vessel wall interaction in flowing blood. Blood 64:1228-1233.

15. Sixma, J. J., K. S. Sakariassen, and P. A. Bolhuis. 1982. The role of factor VIII-von Willebrand factor in adhesion of platelet to the vessel wall. In Pathobiology of the Endothelial Cells. H. L. Nossel and K. Vogel, editors.) Academic Press, Inc., New York. 139-152.
16. Zwaginga, J. J., J. J. Sixma, and P. G. de Groot. 1990. Activation of endothelial cells induces platelet thrombus formation on their matrix: studies of a new in vitro thrombosis model with low molecular weight heparin as anticoagulant. Arteriosclerosis. 10:49-61.

17. Houdijk, W. P. M., P. G. de Groot, P. F. E. M. Nievelstein, K. S. Sakariassen, and J. J. Sixma. 1986. Von Willebrand factor and fibronectin but not thrombospondin are involved in platelet adhesion to the extracellular matrix of human vascular endothelial cells. Arteriosclerosis. 6:24-33.

18. Clark, M. R., N. Mohandas, and S. B. Shohet. 1983. Osmotic gradient ektacytometry: comprehensive characterization of red cell volume and surface maintenance. Blood. 61:899-910.

19. Hardeman, M. R., P. Goedhart, and D. Breederveld. 1987. Laser diffraction ellipsometry of erythrocytes under controlled shear stress using a rotational viscosimeter. Clin. Chim. Acta. 165:227-234.

20. Verhoeven, A. J. M., M. E. Mommersteeg, J. W. N. Akkerman. 1984. Metabolic energy is required in human platelets at any stage during optical aggregation and secretion. Biochim. Biophys. Acta. 800:242-250.

21. Nievelstein, P. F. E. M. Ph G de Groot, P. D'Alessio, F. H. G. Heynen, E. Orlando and J. J. Sixma. 1990. Platelet adhesion to vascular cells: the role of exogenous von Willebrand Factor in platelet adhesion. Arteriosclerosis. 10:462469.

22. Sakariassen, K. S., J. D. Banga, Ph. G. de Groot, and J. J. Sixma. 1984. Comparison of platelet interaction with subendothelium of human renal and umbilical arteries and the extracellular matrix produced by human venous endothelial cells. Thromb. Haemostasis. 52:60-65.

23. Jaffe, E. A., R. L. Nachman, C. G. Becker, and C. R. Minick. 1973. Culture of human endothelial cells derived from umbilical cord veins: identification of morphology and immunologic criteria. J. Clin. Invest. 52:2745-2756.

4. Willems, C., G. C. B. Astaldi, P. G. de Groot, M. C. Janssen, M. D. Consalves, W. P. Zeylemaker, and J. A. van Mourik. 1982. Media conditioned by cultured human vascular endothelial cells inhibit the growth of vascular smooth muscle cells. Exp. Cell. Res. 139:191-197.

25. Baumgartner, H. R., R. Muggli, T. B. Tschopp, and V. T. Turitto. 1976. Platelet adhesion, release and aggregation in flowing blood: effects of surface properties and platelet function. Thromb. Haemostasis. 35:124-138.

26. Clauss, A. 1957. Gerinnungsphysiologische schnell Methode zur Bestimmung des Fibrinogens. Acta Haematol. 17:237-246.

27. Hamer, R. J., J. A. Koedam, N. H. Beeser-Visser, and J. J. Sixma. 1986 Human factor VIII purification from commercial factor VIII concentrate, characterization, identification and radiolabeling. 1986. Biochim. Biophys. Acta. 873:356-366.

28. MacFarlane, D. E., J. Stibbe, E. P. Kirkby, M. B. Zucker, R. A. Grant, and J. McPherson. 1975. A method for assaying von Willebrand factor (ristocetin cofactor). Thromb. Diath. Hemorrh. 34:306-308.

29. Sixma, J. J., and J. Wester. 1977. The hemostatic plug. Semin. Hematol. 14:265-299.

30. Niiya, K., E. Hodson, R. Bader, V. Byers-Ward, J. A. Koziol, E. F. Plow, and Z. M. Ruggeri. 1987. Increased surface expression of the membrane glycoprotein IIb/IIIa complex induced by platelet aggregation: relationship to the binding of fibrinogen and platelet aggregation. Blood. 70:475-483.

31. Meade, T. W., M. V. Vickers, S. G. Thompson, and M. J. Seghatchian 1985. The effect of physiological levels of fibrinogen on platelet aggregation. 1985. Thromb. Res. 38:527-534.

32. Adler, A. J., A. P. Lundin, M. V. Feinroth, E. A. Friedmann, and G. M Berlyne. 1980. Beta-thromboglobulin levels in nephrotic syndrome. Am. J. Med. 69:551-554.

33. Kuhlmann, U., J. Streurer, K. Rhyner, A. von Felten, J. Brinner, and W. Siegenthaler. 1981. Platelet aggregation and beta-thromboglobulin levels in nephrotic patients with and without thrombosis. Clin. Nephrol. 15:229-235.

34. Gurewich, V., B. Lipinsli, and E. Hyde. 1976. The effect of the fibrinogen concentration and the leucocyte count on intravascular fibrin deposition from soluble monomer complexes. Thromb. Haemostasis. 36:605-614.

35. Sagripanti, A., A. Cupisti, M. Ferdeghini, E. Pinori, and G. Barsotti. 1989. Molecular markers of hemostasis activation in nephrotic syndrome. Nephron. 51:25-28.

36. Thompson, W. D., and E. B. Smith. 1989. Atherosclerosis and the coagulation system. J. Pathol. 159:97-106.

37. Ishida, T., and K. Tanaka. 1982. Effects of fibrin and fibrinogen-degradation products on the growth of aortic smooth muscle cells in culture. Atherosclerosis. 44:161-174.

38. Tomura, S., T. Ideura, T. Ida, Y. Osaka, R. Kuriyama, T. Abe, and J. Takeuchi. 1979. Renal vein degradation products in glomerulonephritis. Clin. Nephrol. 12:248-253. 